\title{
Rescuing experience of myasthenia gravis crisis (with 38 patients of clinical analysis)
}

\author{
Guohua Zhang, Chengguo Zhang ${ }^{*}$, Guanglun Zeng
}

Department of Neurology, The First People's Hospital of Foshan, Foshan, China

Email: ${ }^{*}$ zcguo@,fsyyy.com

Received 13 July 2013; revised 15 August 2013; accepted 4 September 2013

Copyright (C) 2013 Guohua Zhang et al. This is an open access article distributed under the Creative Commons Attribution License, which permits unrestricted use, distribution, and reproduction in any medium, provided the original work is properly cited.

\begin{abstract}
Our research was designed to explore the more effective rescuing treatment by summarizing clinical features of the patients with myasthenia gravis crisis. During the course, we carried out a retrospective analysis about clinical data and the course of diagnose and treatment of 38 patients (65 cases) with myasthenia gravis crisis who were in hospital in recent 15 years. As a result, most patients with myasthenia gravis crisis relieved after positive treatment and the mortality was $3.1 \%$. Among lots of treatments, incision of trachea earlier, hormonal therapy, dry treatment and to unite these three treatments were more effective than other treatments. The effective ratio was $100 \%$. In the end, we concluded that to unite incision of trachea, immunosuppressive treatment and dry treatment reasonably can increase the effective ratio of rescuing myasthenia gravis crisis and improve the prognosis of patients obviously.
\end{abstract}

Keywords: Myasthenia Gravis Crisis; Rescuing Treatment; Tracheotomy; Immunosuppressive Treatment; Dry Therapy

\section{INTRODUCTION}

Myasthenia gravis crisis (MGC) happened when patients with myasthenia gravis (MG) had severe somasthenia in bulbar muscle and respiratory muscle so as to be unable to discharge secretions and maintain adequate ventilation-function. If it is not solved timely and effectively, life was threatened. Recently, due to the standardization of MGC treatment, the mortality of these patients decreased. In order to compare with different rescue measures of MGC and search for better treatment methods, we summarized the clinical features and rescue experience of 38 patients (65 cases) with MGC who were in hospital in our department at last 15 years.

\footnotetext{
"Corresponding author.
}

\section{DATA AND METHODS}

\subsection{Clinical Data}

Since Jan. 1997 to June 2012, our department treated a total of 38 patients with MGC (65 cases). All patients were diagnosed according to $\mathrm{MG}$ and $\mathrm{MGC}$ diagnostic criteria.

\subsection{Gender, Age and the Frequency of Occurrence of Crisis}

In 38 patients, 10 patients ( 25 cases) were male and 28 patients ( 40 cases) were female. The age of patients with MG who occurred MGC were between 19-year-old and 58-year-old, with an average age of 37.3; The age of male patients were between 29-year-old and 58-year-old, with an average age of 45.2; The age of female patients were between 19-year-old and 50-year-old, with an average age of 34.8. Among 10 male patients, 8 occurred more than one times of crisis; Among 28 female patients, only 10 occurred more than one times of crisis.

\subsection{The Type of Crisis and Time of Crisis Occurrence}

All 38 patients with myasthenia gravis crisis belonged to myasthenia crisis at first, and then 3 of them turned to cholinergic crisis and 2 of them turned to brittle crisis after treatment. The shortest interval of onset to the crisis was 1 day while the longest was 8 years; 27 cases with crisis occurred in 1 year, the average was 11 months. The crisis took place more than 2 times in 18 patients. The interval between two crises was between 33 days and 7 years, an average of 18 months.

\subsection{Factors of Induced and Aggravating Crisis}

In the 65 cases of crisis meeting, 28 cases $(43.1 \%)$ occurred in the lower respiratory tract infection; In addition, other factors include: overwork (6 cases), the misuse of 
hormone ( 5 cases), thymoma operation ( 5 cases), cholinesterase inhibitors (ChEI) misuse (4 cases), neuromuscular junction block the misuse of drugs (3 cases), to stimulate the spirit of ( 3 cases), trauma ( 2 cases), Menstrual Lai Chao (2 cases), pregnancy (2 cases), stream of people (1 case), tuberculosis (1 case) and unknown factors ( 3 cases).

\subsection{Clinical Classification and Treatment of the Surviving Rate}

According to the Osserman classification, among 38 patients with crisis , 3 patients (7.90 percent) belonged to the type I before, 8 patients $(21.05 \%)$ belonged to the type II before, 12 cases $(31.58 \%)$ belonged to the type III before, and 15 cases $(39.47 \%)$ belonged to type IV before. 38 cases of patients give up treatment and 2 cases of death, and the remaining 36 cases of the disease after treatment by the turn for the better, surviving rate of 94.7 percent, 3.1 percent mortality rate crisis.

\subsection{Complications}

Among 38 patients with MGC, 9 suffered from thymoma, 6 suffered from thymus hyperplasia, and 3 suffered from hyperthyroidism. The common complications include: lung infection (16 cases), atelectasis ( 2 cases), heart failure ( 2 cases), myocardial infarction, arrhythmias and seizures (1 case).

\subsection{The Treatment of Crisis}

\subsubsection{Early Tracheotomy, Pressure-Assisted Breathing}

When patients with serious respiratory and bulbar muscular weakness after the application of ChEI or with frequent crisis happened, they should be operated tracheotomy as soon as possible to strengthen the respiratory tract care [1].

\subsubsection{Adrenal Corticosteroid Therapy}

In addition to patients who were unwilling to use hormones or unsuitable to use hormone, other with MGC were treated with dexamethasone with in $10-20 \mathrm{mg} / \mathrm{d}$ or methylprednisolone in $1000 \mathrm{mg} / \mathrm{d}$ by intravenous drip for 3 - 5 days. And then the dose of hormones should be reduced until they were treated with prednisone or methylprednisolone tablets by oral. The common side effects should be avoided during treatment.

\subsubsection{Application of ChEI}

Patients who agreed with tracheotomy could be stopped offering ChEI for 72 hours when they were under pressure-assisted breathing, which was called "dry therapy" [2].

\subsubsection{Other Immunosuppressive Treatment Treatments}

There were other treatments including immunosuppressor, gamma globulin [3] and plasma exchange therapy [4].

\subsection{Statistical Methods}

Clinical data of each group compared with the efficient was analysed by $\chi^{2}$ test.

\section{RESULTS}

\subsection{Tracheotomy or Not with the Effect (Table 1)}

In 42 cases with tracheotomy, 40 became better, while in 23 cases without tracheotomy, only 17 cases became better.

\subsection{Hormones Therapy or Not with the Effect (Table 2)}

In 55 cases with hormone therapy, 51 became better, while in 10 cases without hormone therapy, only6 cases became better.

\subsection{A Variety of Treatment between the Effects (Table 3)}

All 40 cases with tracheotomy, hormone therapy and dry therapy became better in the end, while only 17 in 25 cases with other treatments became better.

Table 1. Comparison of efficacy of tracheotomy.

\begin{tabular}{cccc}
\hline Group & Cases & Effective & Efficient \\
\hline Tracheotomy group & 42 & 40 & $95.2 \%$ \\
Non-tracheoto group & 23 & 17 & $73.9 \%$ \\
\hline$\chi^{2}=6.266 ; \mathrm{P}<0.05$. & & &
\end{tabular}

Table 2. Comparison of efficacy of hormone application.

\begin{tabular}{cccc}
\hline Group & Cases & Effective & Efficient \\
\hline Hormone group & 55 & 51 & $92.7 \%$ \\
Non-hormone group & 10 & 6 & $60.0 \%$ \\
\hline$\chi^{2}=8.407, \mathrm{P}<0.05$. & & &
\end{tabular}

Table 3. Comparison of efficacy of other treatments.

\begin{tabular}{cccc}
\hline Group & Cases & Effective & Efficient \\
\hline Tracheotom + Hormone + Dry & 40 & 40 & $100.0 \%$ \\
Other treatments & 25 & 17 & $68.0 \%$ \\
\hline$\chi^{2}=14.565, \mathrm{P}<0.05$. & & &
\end{tabular}




\section{DISCUSSION}

According to relevant report recently, the morbidity of MGC in MG patients was between 8.7 percent and 22.1 percent, and the motality was between $13.2 \%$ and $29.7 \%$ $[5,6]$. In our research, female patients accounted for 73.7 percent. Most of male patients suffered crisis after 40 years old, while most of female patients suffered crisis after 30 years old. Male patients were more serious and easy to recur than female patients $(80.0$ percent of men recured while 35.7 percent of women recured). Most cases belonged to Osserman type III and type IV, and crisis mainly occured in the first year. There were more male patients with thymoma, and the most common complication was lung infection; and mortality in patients with crisis of this article is about 3.1 percent.

The results of our research are consistent with the datas of relevant reports at China and abroad [7,8]. But the biggest difference is that the mortality of MGC in our research was obviously lower than other reports. As a result, we summarize 3 points following to improve our efficiency of rescue patients with MGC.

1) Respiratory control and maintain an effective ventilation

Clear airway and control of breathing is one of the key steps to rescue patients with MGC. The following indications of the tracheotomy are vital: a) respiratory rate increase to more than two times of the normal range; $b$ ) thoracic breathing become weak or disappear while abdominal wall controdiction to breathe happen; c) respiratory secretions increase and can not be discharged by their own; d) the signs of hypoxemia or respiratory failure appear, and blood gas analysis showed hypoxemia and carbon dioxide retention [8]. In addition, we found that most of the crisis in patients with bulbar muscular paralysis and aggravating respiratory muscle paralysis needed tracheotomy, which reminds us that it is necessary to pay attention to the progress of patients with bulbar muscular paralysis. If necessary, early preventive tracheotomy would help control the disease [7].

2) Emphasis on immunosuppressive therapy

Immunosuppressive treatment is mainly directed against the pathogenesis of MG treatment, which inhibiting the T lymphocyte cells so as to reduce the acetylcholine receptor antibody titers and promote the neuromuscular junction function [9]. The most commonly used immunosuppressive treatment is hormone therapy. In our research, the rational use of hormones benefit more patients. 4 points following should be payed attention to in the process of using hormones: a) early application of large-dose hormone get more benefits; b) ChEI could be stopped when large-dose hormone was applicating and breathing was under control; c) the gradual reduction of hormone; d) keep small-dose hormone for a long term, which is possible to prevent the development of the disease [10]. In addiction, other immunosuppressive therapy and immunosuppressive agents such as gamma globulin, also have a good effect $[11,12]$.

3) The application of $\mathrm{ChEI}$

Dry therapy should be applicated in all patients with MGC, so that the motor end plates could recover soon. And then ChEI will become more effective again [13]. Our research find that dry therapy can save patients with any type of crisis, and it is no longer important to identify the type of crisis when it happens.

Finally speaking, early and reasonable combination of all measures above, especially dry therapy, is useful in reducing mortality in patients with MGC. On the other side, it is necessary to prevent some side effects about this strategy, such as infection aggravated, stress ulcer, relapse and so on. In addition, we should pay attention to the complications and incentives of crisis. Above all, early intervention to lung infection is very important [14]. Penicillins and cephalosporins should be the first choice to control the infection [15]. Furthermore, during the treatment of MGC, we must not only take care of patients prudentially, but also keep their water-electrolyte balance, strengthen support for the symptomatic treatment and encourage them to develop a positive attitude towards this disease [16].

\section{ACKNOWLEDGEMENTS}

In this investigation, most patients were treated in ICU of the first people's hospital of Foshan. And the data recording were kindly undertaken by Dr. Zhou Lixin and Dr. Yu Tieou. The authors are gratefully thanks for their kind efforts and hospitality.

\section{REFERENCES}

[1] Feng, T.-Y. and Zhao, Y.-B. (2003) Myasthenia gravis diagnosis and treatment progress. Institute of Neurological Disorders and Stroke, 10, 190-191.

[2] Rowland, L.P. (1980) Controversies about the treatment of myasthenia gravis. Journal of Neurology, Neurosurgery \& Psychiatry, 43, 644-659.

http://dx.doi.org/10.1136/jnnp.43.7.644

[3] Zhang, M., Yang, M.S. and Xu, J.Z. (2000) Immunotherapy of myasthenia gravis. Immunology and Nerve Chinese Journal of Neurology, 7, 237-241.

[4] Cong, Z.Q. (1994) Myasthenia gravis on the latest progress of the treatment. Practical Chinese Journal of Internal Medicine, 14, 326.

[5] Chen, L.E. and Zhang, C.G. (1992) Myasthenia gravis clinical analysis of 26 cases of crisis. Journal of China Institute of Neurological Disorders, 18, 360-361.

[6] Murthy, J.M., Meena, A.K., Chowdary, G.V. and Naryanan, J.T. (2005) Myasthenic crisis: Clinical features, complications and mortality. Neurology India, 23, 83-88.

[7] Thomas, C.E., Mayer, A.S., Gungor, Y., et al. (1997) 
Myasthenia gravis: Clinical feature, mortality, complications, and risk factors for prolonged intuition. Neurology, 48, 1253-1260. http://dx.doi.org/10.1212/WNL.48.5.1253

[8] Zhang, C.G. and Chen, L.E. (1992) Myasthenia gravis crisis rescue experience. Journal of Clinical Neurology, 5, 92-94.

[9] Zhang, C.G., Chen, L.E. and Liu, W.B. (2001) The observation of efficacy and safety of day meal served prednisone treatment of myasthenia gravis. Journal of Clinical Neurology, 14, 240-241.

[10] Huang, Y.M. (1997) Immunology research of myasthenia gravis. Chinese Journal of Neurology, 30, 114-116.

[11] Xu, J.Z., Yang, M.S. and Li, B.H. (1999) 2385 cases of clinical research with myasthenia gravis. Chinese Journal of Neurology, 3, 347-350.

[12] Hahn, A.F. (2000) Intravenous immunoglobulin treatment in peripheral nerve disorder indications mechanisms of action and sideeffects. Current Opinion Neurology, 13, $575-582$.

http://dx.doi.org/10.1097/00019052-200010000-00012
[13] Richman, D.P. and Agius, M.A. (2003) Treatment of autoimmune myasthenia gravis. Neurology, 61, 16521661.

http://dx.doi.org/10.1212/01.WNL.0000098887.24618.A $\underline{0}$

[14] Richman, D.P. and Agius, M.A. (2003) Treatment principles in the management of autoimmune myasthenia gravis. Annals of the New York Academy of Sciences, 998, 457-472. http://dx.doi.org/10.1196/annals.1254.060

[15] Younqer, D.S. and Raksadawan, N. (2001) Medical therapies in myasthenia gravis. Chest Surgery Clinics of North America, 11, 329.

[16] Liu, C.C., Li, Z.L., Xian, S.M., et al. (2007) Care and emergency treatment of myasthenia gravis crisis. Chinese Journal of Practical Medicine, 2, 117-118. 